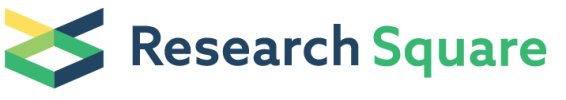 \\ Preprints are preliminary reports that have not undergone peer review. \\ They should not be considered conclusive, used to inform clinical practice, \\ or referenced by the media as validated information.
}

\section{The efficacy of first-line ART regimens based on RPV in HIV- infected patients with pre-existing E138A mutation in reverse transcriptase}

\section{Anna Kuznetsova ( $\square$ a-myznikova@list.ru )}

Gamaleya Centre for epidemiology and microbiology, Moscow, Russia

\section{Aleksey Lebedev}

Gamaleya Centre for epidemiology and microbiology, Moscow, Russia

\section{Konstantin Gromov}

Gamaleya Centre for epidemiology and microbiology, Moscow, Russia

\section{Elena Kazennova}

Gamaleya Centre for epidemiology and microbiology, Moscow, Russia

\section{Maurizio Zazzi}

University of Siena

\section{Francesca Incardona}

I-PRO, Rome

\section{Anders Sönnerborg}

Karolinska Institute

\section{Marina Bobkova}

Gamaleya Centre for epidemiology and microbiology, Moscow, Russia

\section{Research Article}

Keywords: HIV, E138A, reverse transcriptase, RPV.

Posted Date: April 9th, 2021

DOI: https://doi.org/10.21203/rs.3.rs-402978/v1

License: @ (i) This work is licensed under a Creative Commons Attribution 4.0 International License. Read Full License 


\section{Abstract}

Introduction It was previously shown that the presence of the E138A mutation is associated with resistance to rilpivirine (RPV). Detection of this mutation is considered as contraindication for RPV use within the first-line ART. It is a lack of knowledge regarding efficacy of first-line RPV-based ART in patients bearing HIV with E138A mutation. In absence of HIV genotyping in naïve patients it may influence the clinical decisions.

Methods We have collected all available patients unconventionally treated with RPV and carefully analyzed the ART efficacy in E138A carriers from the EuResist database. The viral load data in patients with E138A mutation at baseline was extracted from the database. Due to uniqueness of these cases only $11 \mathrm{HIV}$ infected patients were found. The virologic outcome was analyzed according to the national and international ART guidelines.

Results The full virologic efficacy of the first-line RPV-based ART regimen was demonstrated in 11 out of 11 patients according to all guidelines.

Conclusions Our data suggest that the influence of the pre-existing E138A mutation on the sensitivity to RPV is very low or insignificant. The results support importance of investigation of polymorphic pre-existing HIV drug mutations for ART efficacy.

\section{Introduction}

Rilpivirine (RPV), a second-generation non-nucleoside inhibitor (NNRTI), with confirmed efficacy, safety and tolerability [1], is currently approved for the treatment of HIV infection in first-line three-drug antiretroviral therapy and as two-drug (DTG/RPV) maintenance in European guidelines [2]. Recently, the safety and acceptability of the long acting (LA) RPV as a mean of HIV pre-exposure prophylaxis was reported [3]. RPV is also evaluated as LA intramuscular ART included in cabotegravir/RPV [4], which makes it possible to expand the use of RPV in the future.

The HIV Drug Resistance Stanford database defines E138A as a polymorphic mutation weakly associated with reduced susceptibility to etravirine (ETR) and rilpivirine (RPV) [5], whereas HIV French Resistance database (ANRS) defines the E138A virus as fully resistant to RPV [6]. In in vitro experiments, E138A decreases the susceptibility to RPV about 2-fold [7].

Globally, in antiretroviral therapy (ART)-naïve patients the prevalence of the mutations at E138 varies significantly by the HIV-1 subtype and is highest for subtype C (6.1\%), F (5.1\%), and A (3.3\%) [8]. The variant of HIV-1, sub-subtype A6, that caused the epidemic in Russia, induces for more than $70 \%$ of HIV-infection cases in Russia now $[9,10]$. The prevalence of this polymorphic mutation in A6 viruses in Russia is 4-8\%, depending on the geographical region [10]. Hitherto, E138A has not been included into the surveillance drug resistance mutations (SDRMs) list recommended by the World Health Organization (WHO) for the surveillance of transmitted HIV drug resistance [11]. However, some researchers accounted for this mutation when monitoring the non-nucleoside reverse transcriptase inhibitors (NNRTIs) drug resistance mutations in HIV-1-infected naive patients $[12,13,14,15]$.

Machnowska et al. noted a high prevalence of NNRTIs mutations at polymorphic position E138 and suggested that the alternative first-line regimens combining two NRTIs (nucleoside reverse transcriptase inhibitors) with dolutegravir (DTG) should be preferred [14]. Theys et al. confirmed that, in the geographical regions with high E138A prevalence, RPV-based first-line ART could be compromised when initiated in the absence of routine genotypic resistance testing (GRT) in treatment-naive patients [16]. Furthermore, presence of E138A mutation could impact treatment efficacy or prevention strategies that include RPV in geographical areas where the subtype $\mathrm{C}$ infection is prevalent [17]. Thus, in the countries where HIV GRT is performed before ART start in all patients, detection of E138A advises against RPV use as part of the first regimen. According to ART guidelines in Russia, HIV genotyping at baseline is not obligatory [18]. 
Since the influence of E138A singletons on RPV activity as part of the first-line regimens is not completely clear, the aim of this study was to analyze the virologic efficacy of the first-line ART regimen based on RPV in HIV-1 infected patients with pre-existing E138A mutation in reverse transcriptase. It is very rare that HIV patients with this mutation are treated with RPV since it is against existing recommendations. Therefore we found only 11 patients with such a history of ART in all EuResist database. Nevertheless, the results of such an unconventional therapy may be of interest to both clinicians and virologists studying the phenomenon of HIV drug resistance.

\section{Results}

After database mining 11 participants were included in the study: 8 men and 3 women, with the median age 35.5 and with viral load at baseline from 2625 to 57500 copies/mL. Six patients were infected by HIV-1 subtype B, three - by HIV-1 subtype C, one - by HIV-1 subtype A1 and one - by HIV-1 CRF02_AG.

The follow-up periods and frequency of VL measuring varied from 78 to 209 weeks and from 5 to 14 times, respectively (Table I).

According to the database data of VL measurement at week 4 from ART start were available for 8 out of 11 patients. In these 8 patients, VL decreased by more than $1 \mathrm{lg}$ (Table I), which corresponds to the first criterion of effective therapy in Russian ART guidelines.

We found that 12 weeks ( 3 months) from the ART start, VL was below 400 copies/mL in 10 out of 11 patients. In a patient with the highest VL at baseline (449488), data at 12 week were absent. Interestingly, VL in this patient at 4.5 weeks from ART start decreased down to 999 copies/ml. However, the results of VL measuring in 10 out of 11 patients fully corresponded to the second criterion of efficient therapy in Russian ART guidelines.

We also observed that after 24 weeks from the ART start, all eleven patients had VL below 200 copies/mL (Table I). According to European guidelines this value corresponds to the absence of incomplete suppression. Following DHHS (U.S. Department of Health and Human Services) ART guidelines, this value indicates the absence of virological failure according to one of two criteria. At week 24 from the ART start 10 out of 11 patients had VL below $50 \mathrm{copies} / \mathrm{mL}$ (Table I) and in one patient, 502672, the VL decreased at week 10 down to 147 RNA copies/ml and at week 40 it was below 50 copies/ml. This result was also classified as a virological response.

Therefore, according to the Russian ART guidelines, data for all eleven patients were consistent with the third criteria of efficient therapy.

According to our data all patients demonstrated undetected VL within weeks 3 to 40 (Table I). In all 11 patients, the sustained viral suppression below detection level $(<50 \mathrm{copies} / \mathrm{mL})$ was observed throughout the entire period of observation (Figure I). These VL values correspond to the absence of virological failure according to the Russian guidelines. In European and DHHS guidelines, they correspond to the absence of virological failure, according to the second criterion.

\section{Discussion}

Despite the obvious benefits of ART, the emergence of drug resistance mutations in HIV genome can severely compromise its long-term efficacy. The possibility of transmission of resistant viral strains and infecting with resistant HIV viruses poses additional challenges for treatment and can compromise public ART programs in settings that use standardized first-line regimens. However, mutations in positions associated with resistance are not always a consequence of treatment or transmission of resistant viruses but may reflect the phenomenon of natural polymorphism and have a subtype-specific 
nature resulting from the founder effect during the evolutionary formation of subtypes. The knowledge of the impact of such pre-existing mutations on phenotypic HIV drug resistance remains very limited.

A literature review showed that there is no consensus on the significance of pre-existing HIV drug resistance mutations, and the separate analysis of genotype and phenotype in ART-naive subjects may provide conflicting results. For example, the analysis of 18 subjects with minor resistance mutations in HIV-1 protease at baseline showed no signs of clinical resistance during the ongoing ART [19]. No association was found between pre-existing resistance alone to NNRTIs and rates of virological suppression for patients receiving an efavirenz/emtricitabine/tenofovir regimen [20]. It was also shown that antiviral activity of INSTIs (integrase strand transfer inhibitors) was retained in HIV-1 mutants with minor resistanceassociated mutations [21]. Although it is a deficit of information regarding mutation E138A we found several publications on this issue. In the SPIRIT Study none of the virologically suppressed patients with single E138A mutation had virological failure through week 48 after switching to rilpivirine/emtricitabine/tenofovir disoproxil fumarate [22]. In a study in patients entering the SENSE trial of first-line ART in Europe, Russia and Israel, $13.9 \%$ of patients had one polymorphic mutation in baseline plasma (V90I, V106I or E138A), without any impact on virological outcomes of etravirine-based ART [23]. In a South African cohort, detection of drug-resistant mutations alone including E138A did not predict an increased risk of virological failure [24]. Additionally, in the Eviplera clinical trial there was one patient with mutation E138A at baseline, which maintained virological suppression throughout week 48 [25].

Alternative to these findings, an opinion exists that polymorphic mutations are not fully indifferent for the ART effectiveness and may constitute a kind of support for major resistance mutations. In this role, they can compensate for deleterious effect of major mutations, accelerate their selection, influence the time to resistance development, and the choice of mutational pathways [26].

This is well established that polymorphisms at sites associated with HIV drug resistance occur frequently [27], and the attitude to this issue is different in different countries. In countries where HIV genotyping is routinely performed prior to ART initiation, the presence of any mutations in positions associated with drug resistance ultimately prevents using the appropriate drugs. For example, according to the RPV package insert [28], the presence of E138A prior to therapy may reduce the antiviral activity of RPV, and, in Europe, RPV-based ART will be not recommended to patients with this mutation.

In countries where HIV GRT is not performed before starting treatment, the widespread occurrence of such mutations could potentially lead to ART failure in a significant number of first-line patients. Due to this, it is believed that there is a higher risk of virological failure of RPV-based ART in geographic regions such as sub-Saharan African in which HIV- 1 subtype C infections predominate (that is, E138A mutation is widespread) [29]. We observed similar issue in Russia, where the frequency of the E138A polymorphic mutation in patients infected with the dominant HIV A6 virus ranges from 4 to $8 \%$. This mutation is not associated with the transmission of resistant viruses, since the frequency of its occurrence before the use of RPV and ETR in Russia (until 2011) differed marginally from that in the subsequent time period [30].

Since it is planned to expand the use of RPV in Russia, including as part of single-tablet regimens, we were faced with a particular question, namely, whether patients with pre-existing E138A mutation are more prone to rapid failure of first-line RPV-based ART. To explore this issue, we undertook a search for RPV-treated HIV patients in the presence of E138A mutation. Since in Russia GRT is not performed before starting ART, we had to look for such cases outside the country, namely in the largest European database of HIV genotypes EuResist. The data on viral load during the time of the use of first-line RPV-based ART were analyzed in detail in accordance to Russian and international criteria.

Our study demonstrated that virological efficacy of first-line ART regimen based on RPV in HIV-infected patients with preexisting E138A mutation in reverse transcriptase did not meet the definition of virological failure and corresponded to three out of three criteria of efficient therapy according to the Russian guidelines. At the same time, it did not meet two out of two criteria of virological failure in European and DHHS clinical guidelines. The sustainability of the virological response in all 
patients in this study suggests that a single pre-existing polymorphic E138A mutation unlikely will reduce the effectiveness of RPV-containing first-line regimens.

Currently, the HIV treatment coverage in Russia is increasing and in 2018 the locally produced generic form of RPV (Lakonivir) was registered. In the absence of the routine HIV GRT before starting ART our results may build background for clinical solutions for prescribing first-line RPV. Our findings provide additional information on the effect of single preexisting mutations in positions associated with resistance on the ART effectiveness and advocate for expanding studies of this kind.

Our study has several limitations. Only very small number of patients was observed with different observation time. This is retrospective study with the measures of VL and patient examination not been scheduled consistently. Additionally, the HIV patients were infected with different non-A6 HIV-1 subtypes viruses. Investigation of E138A mutation in HIV A6 sub-subtype is pending.

In conclusion, in our investigation of ART naive patients the singleton pre-existing E138A mutation did not result in failure of RPV-based ART. The exact role of this mutation and other singletons in the efficacy of a first-line ART regimens should be clarified and merits further investigation.

\section{Methods}

\section{Study design and participants}

The EuResist Integrated DataBase (EIDB) (https://www.euresist.org/) was queried for patient cases satisfying the following criteria: a) age over 18 years, b) RPV as part of the first line ART regimen, c) regimen maintenance for more than 40 weeks, d) E138A mutation at baseline and absence of other drug resistance RPV mutations, and e) absence of major NRTIs mutations.

Patients were followed at Italian and Swedish national clinical centers participating to the EuResist Network and provided written informed consent. Ethics approval is not applicable for this study.

The analysis of ART virological outcome was based on the definition of effective therapy and virological failure, according to the Russian national guidelines, and the definition of virological failure, according to European and Department of Health and Human Services (DHHS) clinical guidelines [2, 18, 31], as follows:

1. The viral load (VL) decreased by one log or more at week 4 after the ART start. First criterion of effective therapy according to Russian national guidelines [18].

The results of VL measuring at week 4 were analyzed; alternatively, the results of VL measuring within the time interval of 2 to 4 weeks from ART start, closest to week 4.

2. The VL decreased at week 12 after the ART start below 400 RNA copies/ml. Second criterion of effective therapy according to Russian national guidelines [18].

The results of VL measuring in the time interval from 2 to 12 weeks from ART starting, closest to week 12 were analyzed.

3. The analysis of VL at week 24 after the ART start and its evaluation compared to the two reference HIV-RNA values:

- 200 copies/ml. VL > 200 copies/ml at week 24 after starting therapy in people living with HIV (PLWH), not previously on ART according to the European clinical guidelines is defined as incomplete suppression and is the first criteria of virological failure [2]. Similarly, according to the DHHS guidelines, the inability to achieve $V L<200 \mathrm{copies} / \mathrm{ml}$ is the criterion of virological failure [31]. 
- 50 copies/ml. VL $<50$ copies/ml at week 24 from therapy starting corresponds to the third criteria of effective therapy in Russian national guidelines [18].

The results of VL measurement in the time interval from 4 to 24 weeks from ART start, closest to week 24, were analyzed.

4. Availability of information for time period to undetectable $V L$ and the ability to maintain undetectable $V L$ throughout the follow-up period.

In the Russian national guidelines, virological failure is defined as two consecutive VL levels $>50$ copies/mL detected with an interval of 2-4 weeks in PLWH with previously undetectable HIV-VL at 6 months and later after ART start [18].

According to European guidelines, the second criterion of virological failure is VL rebound as confirmed VL $>50 \mathrm{copies} / \mathrm{mL}$ in PLWH with previously undetectable HIV-VL [2].

In DHHS clinical guidelines, the criterion of virological failure is defined as the inability to maintain suppression of viral replication to an HIV RNA level < 200 copies/mL [31].

According to the combination of all three clinical guidelines, the VL level below 50 copies/mL was defined as a cut-off in this study.

\section{Declarations}

\section{Declarations}

\section{Competing interests}

The authors declare that they have no competing interests.

\section{Authors' contributions}

Kuznetsova A.: Methodology, Formal analysis, Visualization, Writing - Original Draft. Lebedev A.: Visualization, Writing Original Draft. Gromov K.: Formal analysis, Visualization. Kazennova E.: Validation, Writing - Review \& Editing. Zazzi M.: Investigation, Resources, Writing - Review \& Editing. Incardona F.: Investigation, Resources, Writing - Review \& Editing. Sönnerborg A.: Investigation, Resources, Writing - Review \& Editing. Bobkova M.: Conceptualization, Methodology, Writing Review \& Editing

\section{Acknowledgements}

The authors acknowledge the contribution of CARE Consortium funded by the European Union's Horizon 2020 research and innovation program under grant agreement No 825673 and the Ministry of Science and Higher Education of the Russian Federation (unique project identifier RFMEFI61019X0020). The team of authors also acknowledges assistance from medical writers, proof-readers and editors.

\section{References}

1. Bagella, P. et al. Durability, safety, and efficacy of rilpivirine in clinical practice: results from the SCOLTA Project. Infect Drug Resist. 11, 615-623 https://doi.org/10.2147/IDR.S152090 (2018).

2. EACS European AIDS Clinical Society Guidelines Version 10.0 November 2019 https://www.eacsociety.org/files/2019_guidelines-10.0_final.pdf, accessed April 05, 2021. 
3. Cranston, R. D. et al. A Multiple Dose Phase 1 Assessment of Rilpivirine Long Acting in a Model of Preexposure Prophylaxis Against HIV. AIDS Res Hum Retroviruses. 35 (9), 794-804 https://doi.org/10.1089/AID.2018.0265 (2019).

4. Margolis, D. A. et al. Long-acting intramuscular cabotegravir and rilpivirine in adults with HIV-1 infection (LATTE-2): 96week results of a randomised, open-label, phase2b, non-inferiority trial. Lancet. 390 (10101), 1499-1510(2017). doi:10.1016/S0140-6736(17)31917-7.

5. Standford University HIV drug resistance database. NNRTI resistance notes. https://hivdb.stanford.edu/drsummary/resistance-notes/NNRTI/\#63Haddad 2011, accessed April 05, 2021.

6. ANRS. HIV French Resistance. HIV genotypic drug resistance interpretation's algorithms. Tables of rules. http://www.hivfrenchresistance.org/2019/tab3.html, accessed April 05, 2021.

7. Xu, H. T. et al. Effect of mutations at position E138 in HIV-1 reverse transcriptase and their interactions with the M184I mutation on defining patterns of resistance to nonnucleoside reverse transcriptase inhibitors rilpivirine and etravirine. Antimicrob Agents Chemother. 57 (7), 3100-3109 https://doi.org/10.1128/AAC.00348-13 (2013).

8. Calvez, V. et al. Systematic review to determine the prevalence of transmitted drug resistance mutations to rilpivirine in HIV-infected treatment-naive persons. Antivir Ther. 21 (5), 405-412 https://doi.org/10.3851/IMP3024 (2016).

9. Lapovok, I. et al. Molecular epidemiological analysis of HIV-1 genetic variants circulating in Russia in 1987-2015. Terapevtichskij arkhiv. 89(11), 43-48 (in Russian)(2017). https://doi.org/10.17116/terarkh2017891144-49

10. Kazennova, E. V., Lapovok, I. A., Laga, V., Ye, Vasilyev, A. V. \& et Bobkova, M. R. Natural polymorphisms of HIV-1 IDUA variant pol gene. HIV-infection and immune disorders. 4, 44-51 (in Russian) (2012).

11. Bennett, D. E. et al. Drug Resistance Mutations for Surveillance of Transmitted HIV-1 Drug-Resistance: 2009 Update. PLoS One. 4 (3), (2009). e472

12. Calvez, V. et al. Systematic review to determine the prevalence of transmitted drug resistance mutations to rilpivirine in HIV-infected treatment-naive persons. Antivir Ther. 21 (5), 405-412 https://doi.org/10.3851/IMP3024 (2016).

13. Alvarez, M. et al. Transmitted drug resistance to rilpivirine in newly diagnosed antiretroviral naive adults. Clin Microbiol Infect. 21(1), 104.e1-5(2015). doi: 10.1016/j.cmi.2014.08.005.

14. Machnowska, P. et al. Prevalence and persistence of transmitted drug resistance mutations in the German HIV-1 Seroconverter Study Cohort. PLoS One. 14 (1), e0209605 https://doi.org/10.1371/journal.pone.0209605 (2019).

15. Rhee, S. Y. et al. Trends in the Molecular Epidemiology and Genetic Mechanisms of Transmitted Human Immunodeficiency Virus Type 1 Drug Resistance in a Large US Clinic Population. Clin Infect Dis. 68 (2), 213-221 https://doi.org/10.1093/cid/ciy453 (2019).

16. Theys, K. et al. Sub-Epidemics Explain Localized High Prevalence of Reduced Susceptibility to Rilpivirine in TreatmentNaive HIV-1-Infected Patients: Subtype and Geographic Compartmentalization of Baseline Resistance Mutations. AIDS Res Hum Retroviruses. 32 (5), 427-433 https://doi.org/10.1089/aid.2015.0095 (2016).

17. Sluis-Cremer, N. et al. E138A in HIV-1 reverse transcriptase is more common in subtype C than B: implications for rilpivirine use in resource-limited settings. Antiviral Res. 107, 31-34 https://doi.org/10.1016/j.antiviral.2014.04.001 (2014).

18. Clinical guidelines of the Ministry of Health of the Russian Federation. HIV infection in adults. 2020. In Russian.

19. Gehringer, H., Bogner, J. R., Goebel, F. D., Nitschko, H. \& von der Helm, K. Sequence analysis of the HIV-1 protease coding region of $18 \mathrm{HIV}-1$-infected patients prior to HAART and possible implications on HAART. J Clin Virol. 17 (2), 137-141 https://doi.org/10.1016/s1386-6532(00)00086-x (2000).

20. Derache, A. et al. Impact of Next-generation Sequencing Defined Human Immunodeficiency Virus Pretreatment Drug Resistance on Virological Outcomes in the ANRS 12249 Treatment-as-Prevention Trial. Clin Infect Dis. 69 (2), 207-214 https://doi.org/10.1093/cid/ciy881 (2019).

21. Margot, N. A., Ram, R. R., White, K. L., Abram, M. E. \& Callebaut, C. Antiviral activity of HIV - 1 integrase strand - transfer inhibitors against mutants with integrase resistance - associated mutations and their frequency in treatment - naïve 
individuals. J Med Virol. 91 (12), 2188-2194 https://doi.org/10.1002/jmv.25564 (2019).

22. Porter, D. P. et al. Clinical Outcomes of Virologically-Suppressed Patients with Pre-existing HIV-1 Drug Resistance Mutations Switching to Rilpivirine/Emtricitabine/Tenofovir Disoproxil Fumarate in the SPIRIT Study. HIV Clin Trials. 17 (1), 29-37 https://doi.org/10.1080/15284336.2015.1115585 (2016).

23. Geretti, A. M. et al. Sensitive testing of plasma HIV-1 RNA and sanger sequencing of cellular HIV-1 DNA for the detection of drug resistance prior to starting first-line antiretroviral therapy with etravirine or efavirenz. J Antimicrob Chemother. 69 (4), 1090-1097 https://doi.org/10.1093/jac/dkt474 (2014).

24. Li, J. Z. et al. Impact of pre-existing drug resistance on risk of virological failure in South Africa. J Antimicrob Chemother. https://doi.org/10.1093/jac/dkab062 (2021).

25. Assessment report of Eviplera, Committee for Medicinal Products for Human Use (CHMP), 24 October 2013. https://www.ema.europa.eu/en/documents/variation-report/eviplera-h-c-2312-ii-0021-epar-assessment-reportvariation_en.pdf, Accessed April 06, 2021

26. Theys, K., Abecasis, A. B. \& Vandamme, A. M. Future Microbiol. 8 (3), 303-306 https://doi.org/10.2217/fmb.13.10 (2013).

27. Vingerhoets, J. et al. Pre-existing mutations in the rilpivirine Phase III trials ECHO and THRIVE: prevalence and impact on virological response. Antivir Ther. 18 (2), 253-256 https://doi.org/10.3851/IMP2358 (2013).

28. Edurant Highlights of prescribing information. https://www.janssenlabels.com/package-insert/productmonograph/prescribing-information/EDURANT-pi.pdf, Accessed April 06, 2021

29. Sluis-Cremer, N. et al. E138A in HIV-1 reverse transcriptase is more common in subtype C than B: implications for rilpivirine use in resource-limited settings. Antiviral Res. 107, 31-34 https://doi.org/10.1016/j.antiviral.2014.04.001 (2014).

30. Kirichenko, A. A. et al. Prevalence and structure of HIV-1 drug resistance among treatment naïve patients since the introduction of antiretroviral therapy in the Russian Federation. HIV Infection and Immunosuppressive Disorders. 11 (2), 75-83 https://doi.org/10.22328/2077-9828-2019-11-2-75-83 (2019). (In Russian)

31. Guidelines for the Use of Antiretroviral Agents in Adults and Adolescents with HIV, https://clinicalinfo.hiv.gov/sites/default/files/guidelines/documents/AdultandAdolescentGL.pdf, Accessed April 06, 2021.

\section{Table 1}


Table 1

Design of analysis and VL measurement results

\begin{tabular}{|c|c|c|c|c|c|c|c|c|}
\hline $\begin{array}{l}\text { Patient } \\
\text { № }\end{array}$ & $\begin{array}{l}\text { VL at } \\
\text { baseline. } \\
\text { copies/ml }\end{array}$ & $\begin{array}{l}\text { Follow- } \\
\text { up } \\
\text { period, } \\
\text { weeks }\end{array}$ & $\begin{array}{l}\text { The number of } \\
\text { VL } \\
\text { measurements } \\
\text { during the } \\
\text { observation } \\
\text { period }^{1}\end{array}$ & $\begin{array}{l}\text { VL at } 4 \\
\text { weeks, } \\
\text { RNA } \\
\text { copies/ml }\end{array}$ & $\begin{array}{l}\text { VL } \\
\text { decrease } \\
\text { from } \\
\text { baseline } \\
\text { at } 4 \\
\text { weeks } \\
\text { (Lg) }\end{array}$ & $\begin{array}{l}\text { VL at } 12 \\
\text { weeks, } \\
\text { RNA } \\
\text { copies/ml }\end{array}$ & $\begin{array}{l}\text { VL at } 24 \\
\text { weeks, } \\
\text { RNA } \\
\text { copies/ml }\end{array}$ & $\begin{array}{l}\text { Time to } \\
\text { reach } \\
\text { undetectable } \\
\text { VL level, } \\
\text { weeks }\end{array}$ \\
\hline 499976 & 2625 & 149 & 7 & 40 & 1.8 & 40 & 40 & 3 \\
\hline 503470 & 4445 & 106 & 8 & 26 & 2.2 & 26 & 13 & 3 \\
\hline 447257 & 5900 & 78 & 6 & - & - & 0 & 0 & 6 \\
\hline 501784 & 6282 & 111 & 7 & - & - & 40 & 40 & 8 \\
\hline 495772 & 16010 & 165 & 11 & 263 & 1.8 & 37 & 37 & 10 \\
\hline 499502 & 19260 & 188 & 12 & 144 & 2.1 & 32 & 32 & 8 \\
\hline 502672 & 22583 & 90 & 5 & - & - & 147 & 147 & 40 \\
\hline 468009 & 30270 & 209 & 14 & 217 & 2.1 & 217 & 1 & 15 \\
\hline 449293 & 39500 & 209 & 12 & 35 & 3.1 & 35 & 35 & 4 \\
\hline 449693 & 53100 & 136 & 9 & 525 & 2.0 & 266 & 33 & 17 \\
\hline 449488 & 57500 & 179 & 10 & 643 & 2.0 & 999 & 31 & 18 \\
\hline
\end{tabular}

\section{Figures}


A

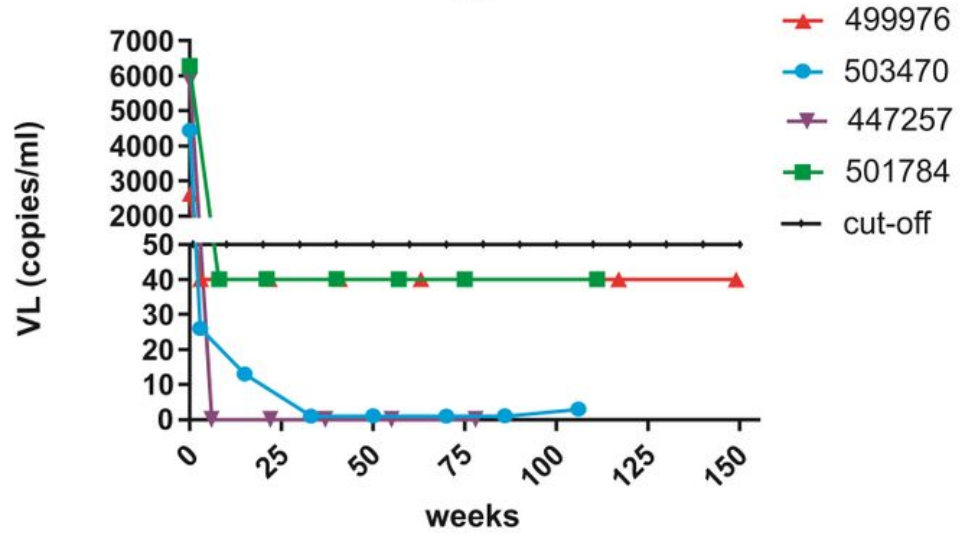

B

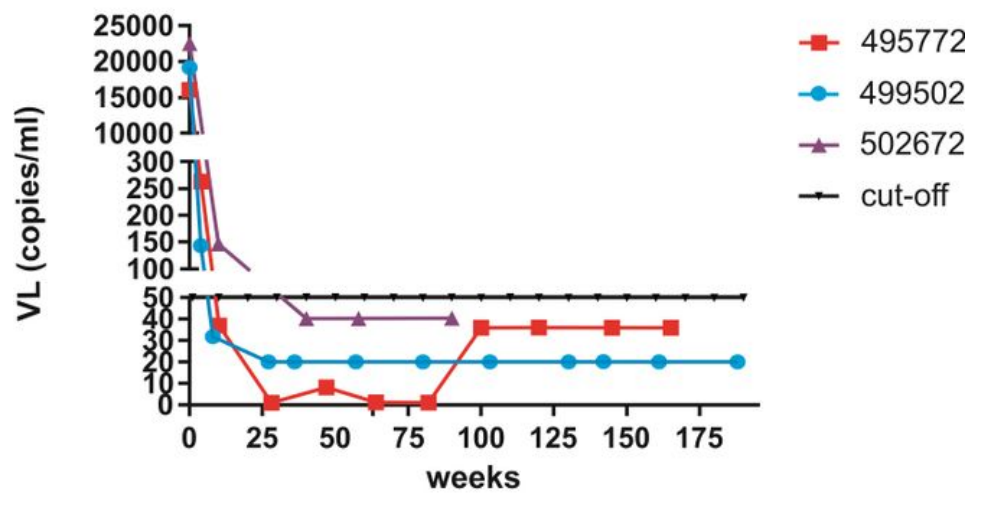

C

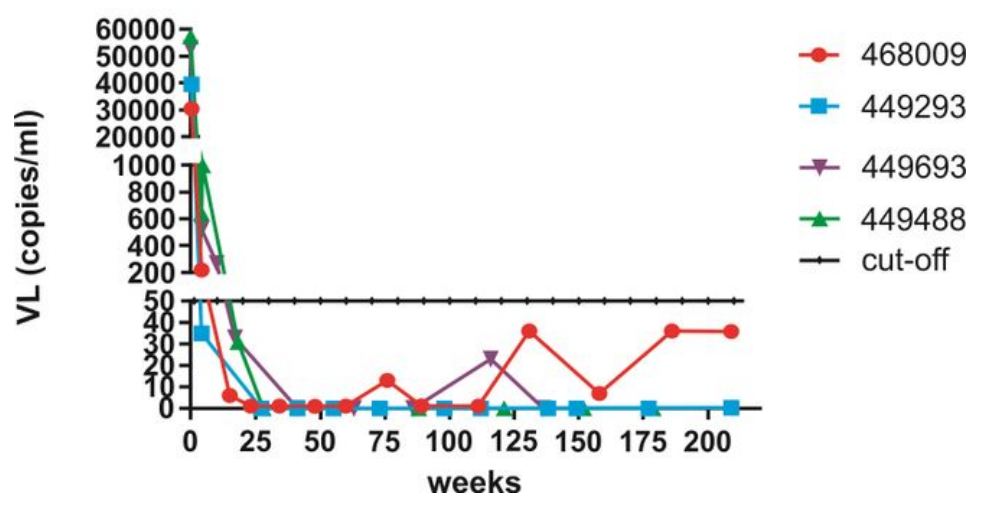

Figure 1

Viral load evaluation (copies/ml) in HIV-infected patients with pre-existing E138A mutation in reverse transcriptase submitted to RPV-based therapy. A - 499976, 503470, 447257, 501784; B - 495772, 499502, 502672; C - 468009, $449293,449693,449438$. 